\title{
The Use of Patient-Reported Outcome Measures in Phase I Oncology Clinical Trials
}

\author{
Robert L. Coleman ${ }^{\mathrm{a}} \quad$ J. Thaddeus Beck ${ }^{\mathrm{b}}$ Joaquina C. Baranda ${ }^{\mathrm{c}}$ Ira Jacobs ${ }^{\mathrm{d}}$ \\ Karen E. Smoyer ${ }^{e}$ Lauren J. Lee ${ }^{d}$ Zemfira Askerova ${ }^{d}$ Justin McGinnis ${ }^{f}$ \\ Apar Kishor Ganti ${ }^{9}$
}

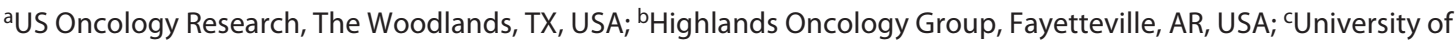

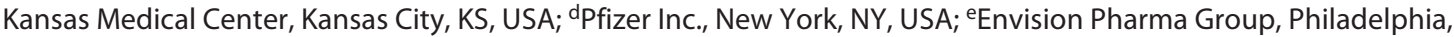
PA, USA; fPfizer Inc., La Jolla, CA, USA; ${ }^{9 V A}$ Nebraska Western lowa Health Care System and University of Nebraska Medical Center, Omaha, NE, USA

\section{Keywords}

Patient-reported outcomes · Phase I - Oncology · Cancer . Clinical trials

\begin{abstract}
Objective: To investigate patient-reported outcome (PRO) usage in phase I oncology clinical trials, including types of PRO measures and changes over time. Methods: We analyzed ClinicalTrials.gov records of phase I oncology clinical trials completed by December 2019. Results: Of all eligible trials, $2.3 \%(129 / 5,515)$ reported $\geq 1 \mathrm{PRO}$, totaling 181 instances of PRO usage. PRO usage increased over time, from $0.6 \%$ (trials initiated before 2000 ) to $3.4 \%$ (trials starting between 2015 and 2019). The most common PRO measures were unspecified (29\%), tumor-specific (24\%), and generic cancer (19\%). Conclusion: Although uncommon in phase I oncology clinical trials, PRO usage is increasing over time. PRO measures were often unspecified on ClinicalTrials.gov, suggesting that more precise reporting and standardization are needed.

(C) 2021 The Author(s)

Published by S. Karger AG, Basel
\end{abstract}

\section{Introduction}

Phase I trials are critically important in drug development and patient care. In oncology, phase I trials traditionally focus on determining the maximum tolerated dose (MTD) and dose-limiting toxicities, as well as accumulating short-term safety (toxicity) and pharmacological data. However, therapeutic intent is becoming an increasingly important focus in phase I oncology trials, with therapeutic intent being a requirement for Medicare coverage of routine costs [1], as well as a recommendation under the American Society for Clinical Oncology policy statement [2]. Consequently, phase I trial designs are evolving to become more adaptive, enabling effective treatments to be approved and made available to patients with cancer more quickly.

Also driving change is the increasing recognition of the value of the patient voice in drug development, through initiatives such as the US Food and Drug Administration (FDA)'s Patient-Focused Drug Development meetings [3]. These meetings are designed to gain patient perspectives on the most important symptoms of their
(C) 2021 The Author(s)

Published by S. Karger AG, Basel

This is an Open Access article licensed under the Creative Commons Attribution-NonCommercial-4.0 International License (CC BY-NC) (http://www.karger.com/Services/OpenAccessLicense), applicable to the online version of the article only. Usage and distribution for commercial purposes requires written permission.
Correspondence to:

Ira Jacobs, ira.jacobs@pfizer.com 
disease, the impacts on daily life, and current treatment approaches. Another way to incorporate the patient voice in drug development is through patient-reported outcomes (PROs) in clinical trials.

The FDA defines a PRO as "any report of the status of a patient's health condition that comes directly from the patient, without interpretation of the patient's response by a clinician or anyone else" [4]. PROs are important because they describe outcomes such as pain, mood, fatigue, psychological and social functioning, and well-being, which are not captured in traditional clinical trial endpoints. Patients can more accurately describe such outcomes than external observers, with physician reports found to commonly underreport symptoms and symptom severity relative to patient reports in a review of oncology clinical trials (including all trial phases) [5], and a study of patients receiving cancer treatment in a real-world setting [6]. The primary importance of collecting PROs is to better understand the patient experience to increase patient centricity and ultimately improve patient care. PROs are also informative study endpoints for the development and evaluation of new drugs [7], as they can measure treatment benefits and risks separately from standard efficacy measures such as response and survival [8]. For these reasons, PROs are of critical importance in clinical trials.

Despite the increasing recognition of the value of patient-centered data, the use of PRO measures in phase I clinical trials remains low [9]. The FDA does not commonly accept PRO data for labeling claims, because PRO measures used in clinical trials are often considered not sufficiently rigorous to warrant a label claim and may be more likely to have missing data compared with clinician-assessed outcomes [10]. In 2009, the FDA issued guidance on incorporation of PROs for measuring disease- or treatment-related symptoms, as well as physical function and health-related quality of life (HRQoL) [4]. FDA guidance was further expanded in 2020 with publication of the patient-focused drug development guidance series [11]. Such expansion of FDA guidance further highlights the growing importance of the patient voice in drug development. PRO data are historically measured in later-phase trials (e.g., phase III). It has been argued that incorporating PROs into earlierphase trials (e.g., phase I or II) would enable PRO measures to be optimized, allowing more rigorous and systematic assessment of PROs in later-phase trials $[12,13]$. These more systematic PRO designs may then increase the chance of a PRO being incorporated into a product's label. Examples of drugs that have achieved PRO-based product label claims include osimertinib for non-small- cell lung cancer $[14,15]$ and ruxolitinib for myelofibrosis $[16,17]$.

Inclusion of PROs in early-phase trials may also differentiate products with similar efficacy but different tolerability profiles, and can also assist in early identification of efficacy and safety profiles, helping to inform the recommended phase II dose (RP2D) [9]. PROs also can provide information on use of healthcare resources and HRQoL for cost-effectiveness analyses in later-phase studies [18], inform payer decision-making, guide questionnaire refinement including psychometric evaluation, and drive patient-focused drug development [19].

The use of HRQoL PROs as endpoints in phase I oncology trials has been examined in the published literature [9]; however, the use of PROs across phase I oncology trials reported on ClinicalTrials.gov and their evolution over time have not been previously described.

This study aimed to conduct a summative assessment of PROs in completed phase I clinical trials of oncology therapies identified in ClinicalTrials.gov, including the proportion of trials incorporating PROs, whether this has been evolving over time, and the types of PROs used in phase I oncology trials, e.g., general HRQoL, symptomspecific PROs, or cancer-specific PROs. We also aimed to put forth recommendations on the potential role and value of PROs in phase I trials for consideration by researchers, clinicians, and patients.

\section{Materials and Methods}

\section{Search Strategy}

Searches were conducted in ClinicalTrials.gov, using a priori defined searches for cancer terms. Eligible trials included adult patients ( $\geq 18$ years of age) with any solid tumor or hematological malignancy. Included within the search results were all interventional trials retrieved by the advanced search function in ClinicalTrials.gov when the trial phase was selected as early phase I or phase I. There were no limits applied to funding source, tumor type, or initiation date; however, trial completion by December 2019 was required. Inclusion criteria defining the population, intervention, comparator, outcomes, and study types (PICOS) are shown in Table 1.

Search results were screened and duplicates removed, along with any studies not meeting the oncology trial inclusion criteria. The study identified phase I oncology trials of interest (the denominator) and the subset of trials that incorporated a PRO (numerator) (shown in Fig. 1).

\section{Data Extraction}

Eligible trials were grouped into 5-year date ranges based on initiation date, with the first group consisting of trials that commenced prior to 2000. The percentage of oncology trials conducted over each time period was calculated for all trials and for trials 
Fig. 1. Clinical trial selection. PRO, patientreported outcome.

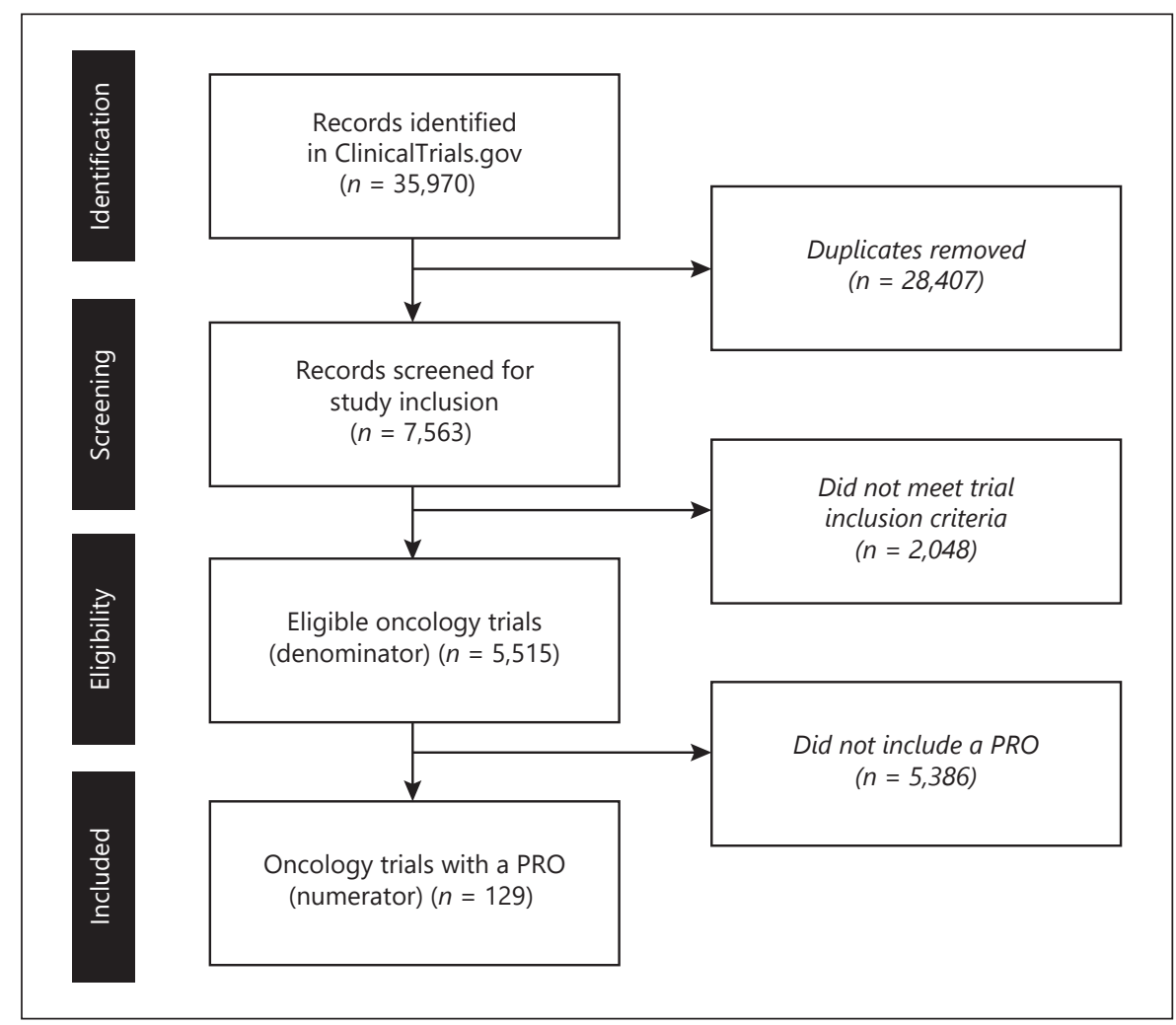

Table 1. Inclusion criteria

\begin{tabular}{ll}
\hline PICOS criteria & Inclusion criteria \\
\hline Population & patients $\geq 18$ years of age, with any solid tumor or hematological malignancy \\
\hline Intervention & $\begin{array}{l}\text { any drug, biologic, or genetic therapy (monotherapy or combination therapy) investigated for the treatment of } \\
\text { cancer, or supportive care agent for chemotherapy-induced neutropenia, anemia, thrombocytopenia, or neuropathy }\end{array}$ \\
\hline Comparator & any or none \\
\hline Outcomes & $\begin{array}{l}\text { for the denominator: any } \\
\text { for the numerator: any PRO including HRQoL and patient-reported symptoms or adverse events }\end{array}$ \\
\hline Study design & completed early phase I, phase I, or phase I/II prospective interventional studies \\
\hline Time period & no time limit other than study completion as of December 2019 \\
\hline
\end{tabular}

HRQoL, health-related quality of life; PICOS, population, intervention, comparator, outcomes, and study types; PRO, patient-reported outcome.

with PROs to track trends over time. Trials with PROs were classified by region (USA only, conducted solely outside of the USA, USA and other countries). Trials with PROs were also grouped by tumor type (including categories for solid tumors - unspecified, and solid plus hematological cancers - unspecified). The five most common tumor types were identified.

For data presented by trial phase, results were categorized by the trial phases listed on the ClinicalTrials.gov downloaded record, which included early phase I, phase I, or phase I/phase II. The definitions of these phases were as per the ClinicalTrials.gov glossary (shown in online suppl. material 1; for all online suppl. material, see www.karger.com/doi/10.1159/000514874) [20].

For each PRO measure, we recorded whether the PRO measure is cancer-specific, the PRO group (defined as generic cancer, generic quality of life [QoL], tumor-specific, mental health, pain, unspecified, other), and whether the PRO measure includes a QoL 
Fig. 2. Cancer trials with $P R O s$ by initiation period. The $n$ values refer to the total number of eligible phase I oncology trials that commenced within the specified time period and were completed by December 2019. PROs, patient-reported outcomes.

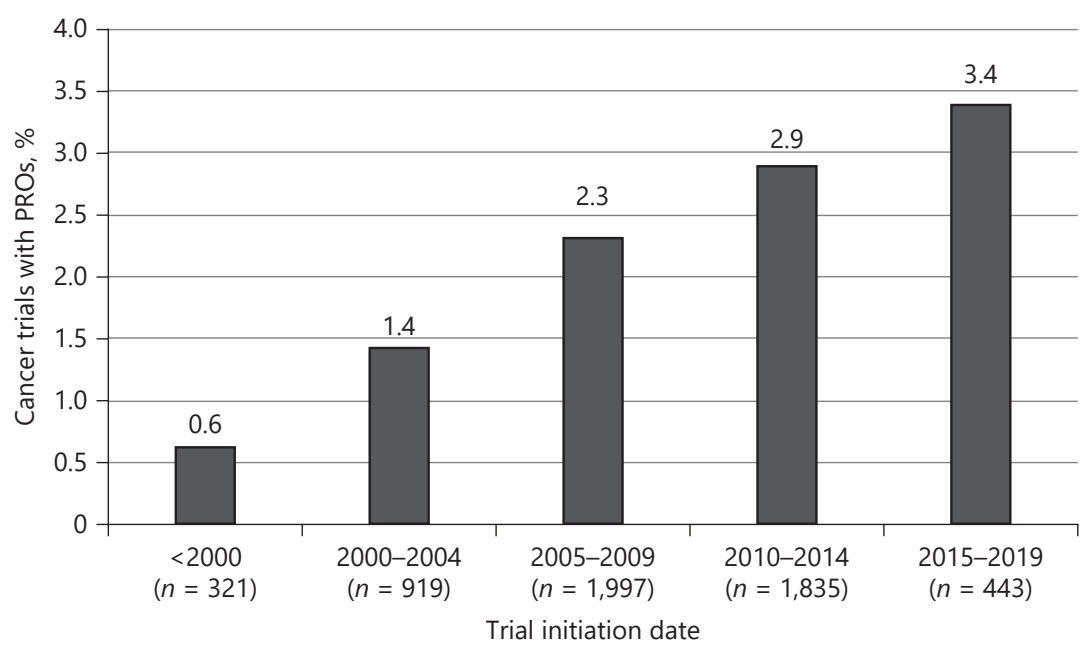

domain (including functional status or well-being), both symptom-specific and QoL domains, symptoms only (no QoL), or a mental health domain.

\section{Results}

\section{Clinical Trial Characteristics}

The search identified a total of 35,970 records, of which 7,563 were screened after removal of duplicates. The denominator consisted of 5,515 eligible phase I oncology trials; 129 of these $(2.3 \%)$ reported one or more PROs (Fig. 1). The total number of instances of PRO usage was 181 (around $30 \%$ of trials reporting PROs used $>1$ PRO measure).

Of the 129 trials reporting a PRO, 64 (50\%) were based in the USA, 48 (37\%) were outside of the USA, 15 (12\%) included locations both within and outside of the USA, and $2(1 \%)$ did not report the location. More than half of the 129 trials received industry funding (64\%).

Among the 5,515 eligible oncology trials, 25\% were combined phase I/phase II trials, $74 \%$ were phase I, and $2 \%$ were early phase I. Of the 129 included trials that reported PROs, $50 \%$ were phase I/phase II, $48 \%$ were phase I, and $2 \%$ were early phase I. Among the trials reporting a PRO, the median trial size was 31 patients (range 1-612).

Across the 129 trials, 25 malignancy types were investigated, the majority of which were solid tumors (82\%). The five most common cancer types were lung (14\%), glioblastoma multiforme (GBM)/brain (11\%), prostate (11\%), pancreatic $(8 \%)$, and liver cancer $(6 \%)$.

Patient-Reported Outcomes in Phase I

Oncology Clinical Trials

\section{Results of PRO Use}

The percentage of trials with PROs, though small, increased steadily over time, from $0.6 \%$ for trials initiated before 2000 to $3.4 \%$ for those starting between 2015 and 2019 (shown in Fig. 2).

A total of 76 unique PRO measures were used, with 52 (68.4\%) of these PRO measures being used only once. The majority of trials with PROs used a single PRO measure (70\%), although one trial included more than four PRO measures. Most PRO measures were secondary endpoints (94\%); only $4 \%$ were primary endpoints (the remaining $2 \%$ were not reported).

Among the top five tumor types in the 129 phase I trials that reported PROs, GBM/brain was the most common tumor type in US-based trials (14\%). Lung cancer was most common in trials conducted solely outside of the US (17\%) or in the US and other countries (20\%) (Fig. 3).

Among specified PRO measures, the most frequent types used were European Organisation for Research and Treatment of Cancer (EORTC; 46 times, 25\%), and Functional Assessment of Cancer Therapy (23 times, 13\%), followed by the Brief Pain Inventory, the MD Anderson Symptom Inventory, and EQ-5D (Fig. 4). Unspecified PRO terms such as "QoL," "pain," or "symptoms" were most commonly used (53 times, 29\%), followed by tumor-specific PRO measures (43 times, 24\%) and generic cancer PRO measures (35 times, 19\%) (Table 2). More than half (54\%) of the 181 PROs included symptom and QoL domains; approximately 30\% reported QoL domains (including unspecified QoL) (Fig. 5). 
Fig. 3. Top five tumors by region in 129 phase I trials with PROs. Ex-USA, conducted solely outside of the USA; GBM, glioblastoma multiforme; PROs, patient-reported outcomes.

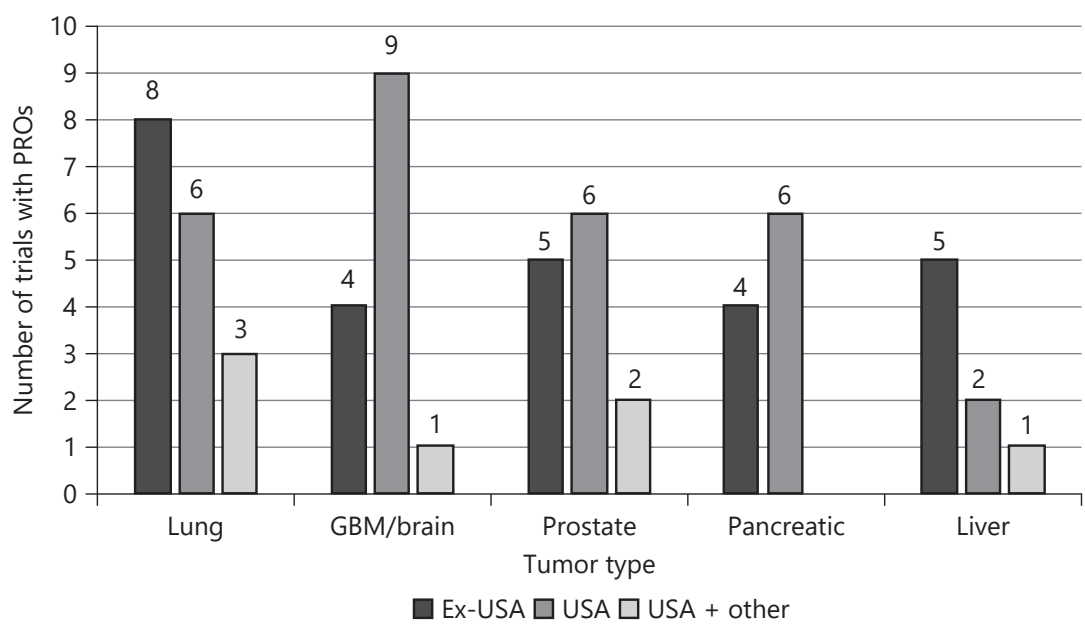

Table 2. Total number of reported PRO measures by trial phase and PRO group

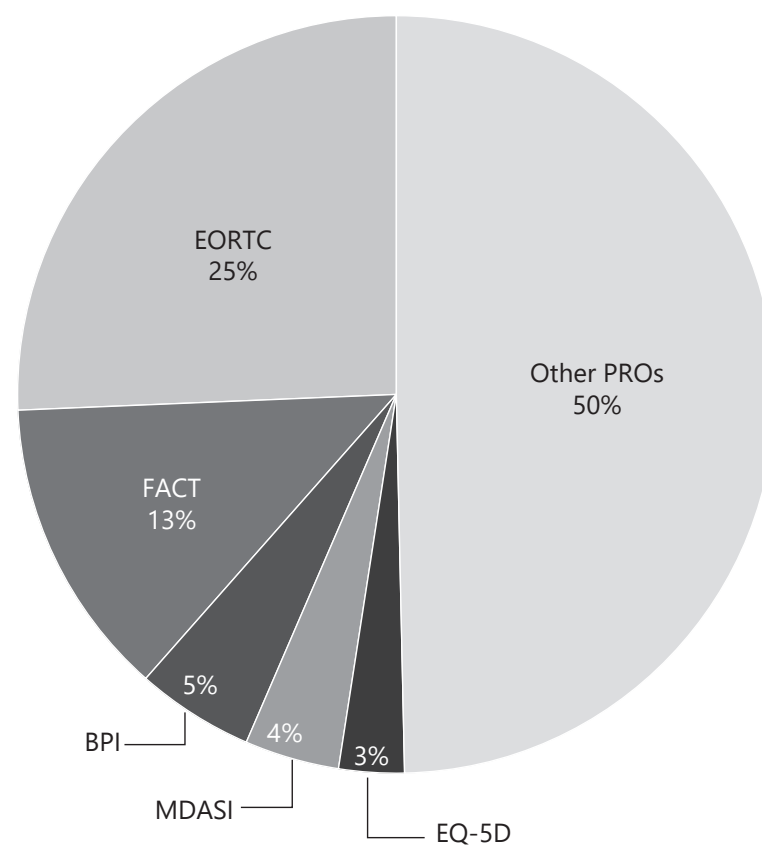

Fig. 4. Frequency of the most commonly used PRO measures in 129 phase I trials. BPI, Brief Pain Inventory; EORTC, European Organisation for Research and Treatment of Cancer; FACT, Functional Assessment of Cancer Therapy; MDASI, MD Anderson Symptom Inventory; PRO, patient-reported outcome.

For lung cancer, the most common PRO measure was the EORTC Quality of Life Questionnaire (EORTC QLQC30, 7/30 PRO measures, 23\%), with the disease-specific module (EORTC QLQ-LC13) also being commonly used

\begin{tabular}{llccc}
\hline PRO group & Early phase I & Phase I & Phase I/II & Total \\
\hline Unspecified & $1(20 \%)$ & $32(37 \%)$ & $20(22 \%)$ & $53(29 \%)$ \\
Tumor-specific & $2(40 \%)$ & $17(20 \%)$ & $24(27 \%)$ & $43(24 \%)$ \\
Generic cancer & 0 & $10(11 \%)$ & $25(28 \%)$ & $35(19 \%)$ \\
Pain & 0 & $10(11 \%)$ & $9(10 \%)$ & $19(10 \%)$ \\
Mental health & $2(40 \%)$ & $9(10 \%)$ & $3(3 \%)$ & $14(8 \%)$ \\
Generic QoL & 0 & $8(9 \%)$ & $4(4 \%)$ & $12(7 \%)$ \\
Other & 0 & $1(1 \%)$ & $4(4 \%)$ & $5(3 \%)$ \\
Total & $5(3 \%)$ & $87(48 \%)$ & $89(49 \%)$ & $181(100 \%)$ \\
\end{tabular}

Values are presented as $n(\%)$. Trial phases are as per the ClinicalTrials. gov record [20]. PRO, patient-reported outcome; QoL, quality of life.

(5/30 PRO measures). For prostate cancer, EORTC QLQC30 was similarly the most common PRO measure (4/24 PRO measures, $17 \%)$, followed by the disease-specific module EORTC QLQ-PR25 (3/24 PRO measures). QoL as an unspecified measure was the most frequently reported PRO measure in the ClinicalTrials.gov record for GBM/brain (35\%), pancreatic (33\%), and liver (33\%) cancer trials. A list of the PRO measures identified, and their frequency of occurrence in the 129 phase I trials, is shown in online supplementary Table S1.

\section{Discussion}

This study confirms and extends prior reports demonstrating that PROs are not commonly incorporated into phase I oncology trials, and suggests that their usage is 
Fig. 5. PROs by domains. PROs, patientreported outcomes; QoL, quality of life.

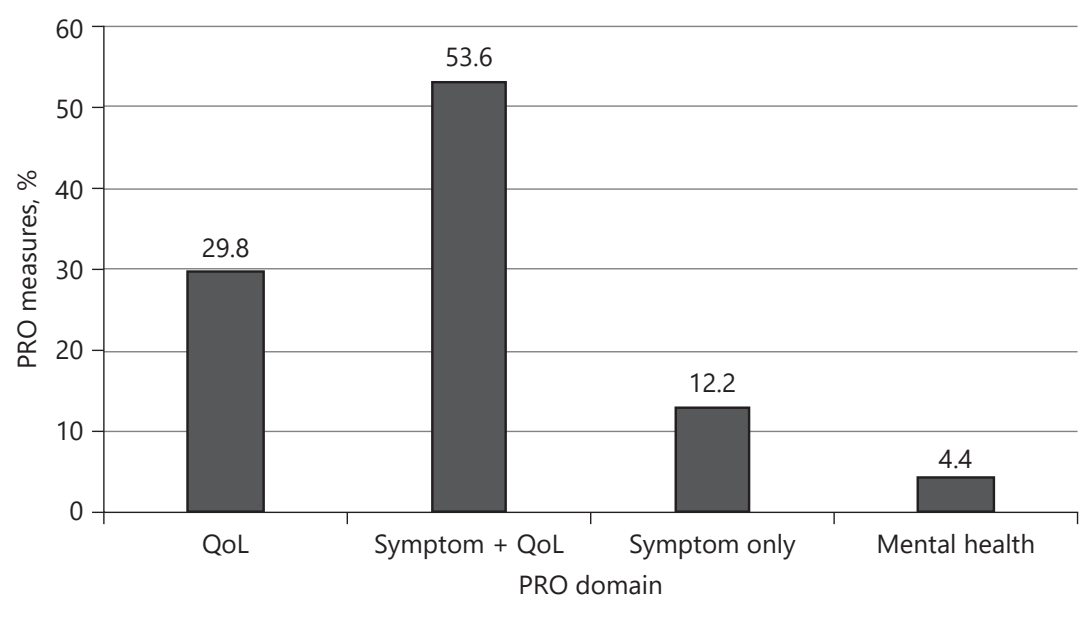

increasing over time. This study highlights a need for more specific and standardized reporting of PRO measures and suggests potential differences in PRO usage depending on region and tumor type.

With increased focus on patient-centered drug development, incorporation of PROs enables inclusion of the patients' perspective on the diverse impacts, both beneficial and harmful, of exposure to investigational agents in early clinical development. PROs in phase I trials can provide early guidance on tolerability which could be used to inform drug development and later-phase trial designs. Recommendations on incorporating PROs in clinical trials are included in key documents, such as American Society of Clinical Oncology recommendations, FDA guidance, and the Standard Protocol Items: Recommendations for Interventional Trials (SPIRIT) statement [2, 4, 21].

This analysis shows that although the use of PROs in phase I trials is limited (averaging 2.3\%), it has been increasing steadily over time from $0.6 \%$ for trials commencing prior to 2000 to $3.4 \%$ for trials commencing between 2015 and 2019. These findings are similar to a previous study based on published literature, which reported that only $1.1 \%$ of phase I oncology trials included an HRQoL PRO endpoint, and none used it to determine RP2D [9]. By contrast, studies across all trial phases and disease types based on ClinicalTrials.gov data have reported higher rates of PRO inclusion, with a study of trials from 2004 to 2007 reporting PRO usage in $14.0 \%$ of interventional trials [22], and a study of trials from 2007 to 2013 reporting PRO usage in $27 \%$ of interventional trials [23]. Both these studies found that phase III trials were most likely to incorporate PRO data [22, 23], consistent with the rate of PRO usage being lower in phase I compared with later-phase trials. This is consistent with our finding that half of the trials with PROs were combined phase I/ II studies, despite this type of trial forming only a quarter of eligible oncology trials in our study. These studies are also consistent with our finding that PRO usage is increasing over time. This increase could be reflective of a growing acknowledgment of the importance of incorporating the patient perspective in drug design and development.

Across the 129 phase I oncology trials identified in this study, we identified 181 instances of PRO usage, with a sizable percentage of clinical trials reporting unspecified PRO measures such as "QoL," "pain," or "symptoms." Similarly, an analysis of interventional trials of any phase registered in ClinicalTrials.gov found that, of trials with a PRO measure, less than half (41\%) identified the instrument to be used [22]. It is unclear whether this is due to the PRO measure not yet having been identified at the time the ClinicalTrials.gov record was created, or whether there may be a perception that PRO endpoints do not require the same degree of specificity as other clinical trial endpoints [21]. This differs from clinical trial reports in peer-reviewed publications, where the instrument used to measure HRQoL PROs was specified in 12/15 phase I oncology publications reporting HRQoL PROs [9]. It is recommended that the choice of PRO measure be carefully considered and documented to optimize data collection and interpretation [21]. These findings highlight the need for more transparency and precision with reporting PRO outcomes in the clinical trial registries.

For clinical trials that reported the PRO measure used, the EORTC QLQ-C30 was the most frequently used PRO 
instrument, consistent with a study of PRO usage in the published literature [9]. Generic cancer instruments such as EORTC QLQ-C30 are advantageous in that they are standardized and validated; however, it is important that these tools be carefully evaluated in the specific target population in the trial. Standard PRO tools and outcome measures may require adjustments to ensure inclusion of symptoms specific to the disease under study, such as using disease-specific modules like LC13 for lung cancer and PR25 for prostate cancer in conjunction with EORTC QLQ-C30, or the PRO version of the Common Terminology Criteria for Adverse Events (CTCAE) [24, 25].

Among the 181 PROs in the 129 trials in this analysis, 78 (43\%) were cancer- or tumor-specific. For trials incorporating PROs, the most common cancer types included lung, GBM/brain, and prostate. For studies based in the US and incorporating a PRO, the most common tumor type was GBM/brain cancer, whereas for studies based outside of the US, the most common tumor type was lung cancer. Further research, which incorporates data on trials not reporting PROs, could investigate any potential associations between cancer subtype, region, and frequency of PRO use. Cancer subtypes could also be compared between trials that did and did not incorporate one or more PRO. As oncology agents become more niched, PRO reporting tools with improved specificity may become increasingly valuable.

Our analysis has a number of strengths and limitations. Strengths include the use of a readily accessible public resource, ClinicalTrials.gov, which is the largest clinical trial registry in the world, with over 360,000 records as of December 2020. Moreover, the information on ClinicalTrials.gov is not subject to publication bias or publication delays. In one analysis, around $50 \%$ of trials on ClinicalTrials.gov were found not to have an associated publication, and of those that did, there was a median time of around 21 months from primary completion to journal publication [26]. Using the ClinicalTrials.gov registry as the data source may therefore allow for a more complete and up-to-date analysis than one from reviews based on published literature. Of note, our analysis did not include trials recorded in other clinical trial registries, such as the World Health Organization International Clinical Trials Registry Platform or the European Union Clinical Trials Register (EUCTR). The ClinicalTrials.gov database may not be representative of all clinical trial registries, and a comparison with the commercial clinical trial database TrialTrove revealed differences between the two databases in clinical trial status, phase, and start year [27]. A key limitation of our study is the potential inclusion of phase II PRO data in our results. For phase I/II trials reporting PROs, ClinicalTrials.gov does not specify whether the PROs were measured during the phase I or phase II portion of the trial. We expect that PROs would more likely be included in the phase II portion rather than phase I. Knowing which phase(s) of these trials incorporated the PRO data is important, as the goals of these trial phases differ, and therefore the types of PRO measures needed may also differ. It is also important to note that PRO measures, where included in clinical trials, may be used as exploratory endpoints rather than primary or secondary outcomes. Exploratory outcomes and post hoc analyses are excluded from the ClinicalTrials.gov record, and so the use of PROs is likely underestimated in this analysis.

Our analysis is reflective of two main ways in which phase I oncology trial designs are evolving. Firstly, although the focus of phase I trials remains on safety and pharmacokinetics/pharmacodynamics, the importance of therapeutic intent in phase I trials is increasingly being recognized, with many phase I trials focusing not solely on determining MTD, but also including efficacy as secondary endpoints [28]. This may reflect a shift in recent years from traditional cytotoxic drugs, for which the MTD typically translates to the RP2D, toward molecularly-targeted agents, immunotherapies, and combination therapies, to which patients may respond at doses below the MTD [29, 30]. Adaptive trial designs, where dosage is adjusted according to efficacy or pharmacodynamic results obtained during the trial, are becoming increasingly common. The use of adaptive trial designs can lead to a more rapid identification of the RP2D and minimize the number of patients receiving doses that are either subtherapeutic or at the MTD, reducing the risk of adverse events [31-34]. Secondly, study designs are increasingly focusing on including information on treatment benefits, toxicities, and outcomes that are meaningful for patients. To accomplish this, investigators are increasingly seeking input from patient advocates during study design, through initiatives such as the FDA's Patient-Focused Drug Development meetings [3]. These changes in trial design may be increasing the need for and reaping the benefits of PROs in phase I trials.

Including PROs in phase I oncology trials can benefit trial sponsors, payers, patients, and clinicians. For trial sponsors, PRO data can differentiate a product and inform payer and healthcare provider decision-making, 
and it is not associated with administration costs other than time constraints for patients and staff. Including PROs earlier in the development process could inform later-phase trials, ensuring that the most appropriate PRO measures are used and improving the robustness of the data obtained in later-phase trials or submitted to regulators for fast tracking or breakthrough designation of new therapies. For example, a PRO-based assessment tool developed during a phase I/II study of the myelofibrosis drug ruxolitinib was used to gather PRO data in the phase III studies, where its efficacy in improving symptoms played a key role in its approval $[16,17]$. In another example, a study of prednisone with or without mitoxantrone for metastatic prostate cancer showed a significant improvement in PROs with mitoxantrone, including pain and global HRQoL, despite no change in overall survival $[35,36]$. These PRO data led to regulatory approval and widespread use of mitoxantrone for metastatic prostate cancer. For patients, including PROs in phase I trials allows them to convey the range of adverse impacts and benefits in addition to researcher-collected clinical response and safety outcomes. This could include impacts or barriers to treatment that are not measurable in the clinic, such as financial toxicity. Inclusion of PROs may empower patients and inform and facilitate patient and clinician treatment decisions.

In order to realize the benefits of including PROs in phase I oncology trials, a number of barriers need to be overcome. Firstly, sponsors have historically had difficulties with PROs being accepted for label claims, which may be a disincentive to include these endpoints in clinical trials. One study showed that of 45 indications that included PRO data in the submission, only 21 received European Medicines Agency PRO labeling, and none received PRO labeling from the FDA [10]. This barrier can potentially be overcome by including PROs earlier in the trial process - such as during phase I trials - providing an opportunity to identify and systematically capture PRO endpoints in later-phase trials. This may increase the robustness of the PRO data, which could assist it in meeting the rigorous standard of evidence required for FDA PRO labeling [4]. Stakeholders could also meet with regulatory bodies and patient advocacy groups to better understand how PROs could be integrated into trial designs. A second barrier would be difficulty in selecting the most appropriate PRO concepts and measures to use in the phase I trial, considering that the dose-limiting toxicities would be unknown early in the trial. As dose escalation nears the MTD, drug treatment may have a greater impact on HRQoL, and adverse events may worsen. Furthermore,
PROs may not be appropriate in studies where many patients are receiving subtherapeutic doses and where there is a very limited number of patients at particular dose levels. A third barrier may be incomplete data resulting from poor completion rates. Care would be needed to ensure that the length and number of PRO measures is kept to a minimum to encourage survey completion. There can also be a perception that PRO endpoints are not as important as clinical endpoints, meaning that researchers may not follow up as carefully with patients. Healthcare providers may be resistant to using PROs or find the number of PRO data points overwhelming [37]. Validation of PRO measures is important in demonstrating PRO suitability and may assist in increasing healthcare providers' acceptance of PRO data. Racial differences in completion rates and preferences for the way patients report PROs [38, 39] may exist as well. These complexities with PRO data interpretation highlight the need for adaptive approaches, such as adaptive trial designs that minimize the number of patients receiving subtherapeutic doses, and PRO tools that can be tailored accordingly as knowledge on treatment toxicities develops.

\section{Conclusions}

This study confirms and extends prior analyses demonstrating that PROs are not commonly incorporated into phase I oncology trials, but suggests that their usage is increasing over time. PROs are expected to be included more frequently in the future as trial designs, regulatory decision-making, and treatment landscapes evolve. This study highlights the need for more precise reporting and standardization of PRO measures used in phase I oncology trials, particularly for PRO reporting in clinical trial registries. Inclusion and standardization of PROs in early-phase trials may improve the designs of later-phase trials, increase the likelihood of PRO claims in the product label, and ultimately empower patients, caregivers, and healthcare providers to make more informed treatment decisions leading to better outcomes and better QoL for patients.

\section{Acknowledgment}

The authors would like to thank Georgina Bowden, $\mathrm{PhD}$, and Catherine Rolland, PhD, of Curo Consulting, Envision Pharma Group, for their assistance with data collection and analysis. Medical writing support was provided by Susan Tan, PhD, of Engage Scientific Solutions, and funded by Pfizer. 


\section{Statement of Ethics}

This research complied with the guidelines for human studies and was conducted ethically in accordance with the World Medical Association Declaration of Helsinki. In this retrospective study, data were collected from published records and thus no patient consent was required.

\section{Conflict of Interest Statement}

R.L. Coleman has received grant funding from AbbVie, AstraZeneca, Clovis, Roche/Genentech, Genmab, Immunogen, Janssen, Merck, and Novartis, and served on scientific steering committees for AbbVie, Agenus, AstraZeneca, Clovis, Genmab, Immunogen, Janssen, Merck, Roche/Genentech, and GlaxoSmithKline. J.T. Beck has received research grant funding from Pfizer, Merck, Nektar, Roche/Genentech, AbbVie, Clovis, Novartis, Immunomedics, Seattle Genetics, AstraZeneca, Eli Lilly, and Daiichi Sankyo, Inc. J.C. Baranda has received research funding from Astellas, Exelixis, Nektar, Pfizer, Sanofi, Tolero, Xencor, SQZ, and owns stock in Forty Seven, Hylapharm, Zymeworks, Moderna, MorphoSys AG, and Aprea Therapeutics. K.E. Smoyer is an employee and shareholder of Envision Pharma Group, which was paid by Pfizer to conduct this study. I. Jacobs, L.J. Lee, and Z. Askerova are employees of and own stock in Pfizer, Inc. J. McGinnis was an employee and stockholder of Pfizer, Inc. at the time this study was conducted. A.K. Ganti has served on advisory boards for Jazz Pharmaceuticals, G1 Therapeutics, Blueprint Medicines, AstraZeneca, Cardinal Health, Flagship Biosciences, has worked as a consultant for AstraZeneca and Genentech, and has received research support from Takeda, Oncoceutics, Merck, AstraZeneca, Novartis, Apexigen, and Nektar. The authors have no other relevant affiliations or financial involvement with any organization or entity with a financial interest in or financial conflict with the subject matter or materials discussed in this paper apart from those disclosed.

\section{Funding Sources}

Not applicable.

\section{Author Contributions}

I. Jacobs, L.J. Lee, J. McGinnis, and K.E. Smoyer contributed to the design, planning, and conception of the study as well as to data interpretation and manuscript development. R.L. Coleman, J.T. Beck, J.C. Baranda, Z. Askerova, and A.K. Ganti contributed to data interpretation and manuscript development. All authors reviewed the manuscript drafts and reviewed and approved the final version for submission.

\section{References}

1 Centers for Medicare \& Medicaid Services. National Coverage Determination (NCD) for routine costs in clinical trials (310.1). 2007 Available from: https://www.cms.gov/medicare-coverage-database/details/ncd-details. aspx?NCDId=1.

2 Weber JS, Levit LA, Adamson PC, Bruinooge $\mathrm{S}$, Burris HA, Carducci MA, et al. American Society of Clinical Oncology policy statement update: the critical role of phase I trials in cancer research and treatment. J Clin Oncol. 2015;33(3):278-84

3 Chalasani M, Vaidya P, Mullin T. Enhancing the incorporation of the patient's voice in drug development and evaluation. Res Involv Engagem. 2018;4:10.

4 US Food and Drug Administration. Guidance for industry. Patient-reported outcome measures: use in medical product development to support labeling claims. 2009. Available from: https: //www.fda.gov/media/77832/download.

5 Xiao C, Polomano R, Bruner DW. Comparison between patient-reported and clinicianobserved symptoms in oncology. Cancer Nurs. 2013;36(6):E1-E16.

6 Basch E, Jia X, Heller G, Barz A, Sit L, Fruscione $\mathrm{M}$, et al. Adverse symptom event reporting by patients vs clinicians: relationships with clinical outcomes. J Natl Cancer Inst. 2009;101(23):1624-32.

7 Fitzpatrick R, Davey C, Buxton MJ, Jones DR. Evaluating patient-based outcome measures for use in clinical trials. Health Technol As sess. 1998;2(14):i-iv, 1-74.

8 Mercieca-Bebber R, King MT, Calvert MJ, Stockler MR, Friedlander M. The importance of patient-reported outcomes in clinical trials and strategies for future optimization. Patient Relat Outcome Meas. 2018;9:353-67.

9 Fiteni F, Ray IL, Ousmen A, Isambert N, Anota A, Bonnetain F. Health-related quality of life as an endpoint in oncology phase I trials: a systematic review. BMC Cancer. 2019;19(1):361.

10 Gnanasakthy A, Barrett A, Evans E, D’Alessio D, Romano CD. A review of patient-reported outcomes labeling for oncology drugs approved by the FDA and the EMA (20122016). Value Health. 2019;22(2):203-9.

11 US Food and Drug Administration. FDA patient-focused drug development guidance series for enhancing the incorporation of the patient's voice in medical product development and regulatory decision making. 2020. Available from: https://www.fda.gov/drugs/ development-approval-process-drugs/fdapatient-focused-drug-development-guidance-series-enhancing-incorporation-patients-voice-medical.

12 Basch E, Dueck AC. Patient-reported outcome measurement in drug discovery: a tool to improve accuracy and completeness of efficacy and safety data. Expert Opin Drug Discov. 2016;11(8):753-8.

13 Wagner LI, Wenzel L, Shaw E, Cella D. Patient-reported outcomes in phase II cancer clinical trials: lessons learned and future directions. J Clin Oncol. 2007;25(32):505862.

14 European Medicines Agency. Assessment report: Tagrisso 2018. Available from: https:// www.ema.europa.eu/en/documents/variation-report/tagrisso-h-c-4124-ii-0019-eparassessment-report-variation_en.pdf.

15 Leighl NB, Karaseva N, Nakagawa K, Cho BC, Gray JE, Hovey T, et al. Patient-reported outcomes from FLAURA: osimertinib versus erlotinib or gefitinib in patients with EGFRmutated advanced non-small-cell lung cancer. Eur J Cancer. 2020;125:49-57.

16 Deisseroth A, Kaminskas E, Grillo J, Chen W Saber H, Lu HL, et al. U.S. Food and Drug Administration approval: ruxolitinib for the treatment of patients with intermediate and high-risk myelofibrosis. Clin Cancer Res. 2012;18(12):3212-7.

17 Verstovsek S, Kantarjian HM, Estrov Z, Cortes JE, Thomas DA, Kadia T, et al. Long-term outcomes of 107 patients with myelofibrosis receiving JAK1/JAK2 inhibitor ruxolitinib: survival advantage in comparison to matched historical controls. Blood. 2012;120(6):12029.

18 Ramsey S, Willke R, Briggs A, Brown R, Buxton $\mathrm{M}$, Chawla A, et al. Good research practices for cost-effectiveness analysis alongside clinical trials: the ISPOR RCT-CEA Task Force Report. Value Health. 2005;8(5):52133. 
19 US Food and Drug Administration. Patientfocused drug development: collecting comprehensive and representative input: guidance for industry, Food and Drug Administration staff, and other stakeholders. 2020. https: //www.fda.gov/media/139088/download.

20 US National Library of Medicine. ClinicalTrials.gov: glossary of common site terms. 2020. Available from: https://clinicaltrials.gov/ct2/ about-studies/glossary.

21 Calvert M, Kyte D, Mercieca-Bebber R, Slade A, Chan AW, King MT, et al. Guidelines for inclusion of patient-reported outcomes in clinical trial protocols: the SPIRIT-PRO Extension. JAMA. 2018;319(5):483-94.

22 Scoggins JF, Patrick DL. The use of patientreported outcomes instruments in registered clinical trials: evidence from ClinicalTrials. gov. Contemp Clin Trials. 2009;30(4):289-92.

23 Vodicka E, Kim K, Devine EB, Gnanasakthy A, Scoggins JF, Patrick DL. Inclusion of patient-reported outcome measures in registered clinical trials: evidence from ClinicalTrials.gov (2007-2013). Contemp Clin Trials. 2015;43:1-9.

24 Kluetz PG, Chingos DT, Basch EM, Mitchell SA. Patient-reported outcomes in cancer clinical trials: measuring symptomatic adverse events with the National Cancer Institute's Patient-Reported Outcomes Version of the Common Terminology Criteria for Adverse Events (PRO-CTCAE). Am Soc Clin Oncol Educ Book. 2016;35:67-73.
25 Dueck AC, Mendoza TR, Mitchell SA, Reeve BB, Castro KM, Rogak LJ, et al. Validity and reliability of the US National Cancer Institute's Patient-Reported Outcomes Version of the Common Terminology Criteria for Adverse Events (PRO-CTCAE). JAMA Oncol. 2015;1(8):1051-9.

26 Riveros C, Dechartres A, Perrodeau E, Haneef R, Boutron I, Ravaud P. Timing and completeness of trial results posted at ClinicalTrials.gov and published in journals. PLoS Med. 2013;10(12):e1001566; discussion e1001566.

27 Stergiopoulos S, Getz KA, Blazynski C. Evaluating the completeness of ClinicalTrials.gov. Ther Innov Regul Sci. 2019;53(3):307-17.

28 Sisk BA, Dubois J, Hobbs BP, Kodish E. Reprioritizing risk and benefit: the future of study design in early-phase cancer research. Ethics Hum Res. 2019;41(6):2-11.

29 Jain RK, Lee JJ, Hong D, Markman M, Gong J, Naing A, et al. Phase I oncology studies: evidence that in the era of targeted therapies patients on lower doses do not fare worse. Clin Cancer Res. 2010;16(4):1289-97.

30 Postel-Vinay S, Arkenau HT, Olmos D, Ang J, Barriuso J, Ashley S, et al. Clinical benefit in phase-I trials of novel molecularly targeted agents: does dose matter? Br J Cancer. 2009; 100(9):1373-8.

31 Le Tourneau C, Gan HK, Razak AR, Paoletti $X$. Efficiency of new dose escalation designs in dose-finding phase I trials of molecularly targeted agents. PLoS One. 2012;7(12):e51039.

32 Ji Y, Wang SJ. Modified toxicity probability interval design: a safer and more reliable method than the $3+3$ design for practical phase I trials. J Clin Oncol. 2013;31(14):178591.
33 LoRusso PM, Boerner SA, Seymour L. An overview of the optimal planning, design, and conduct of phase I studies of new therapeutics. Clin Cancer Res. 2010;16(6):1710-8.

34 Hollebecque A, Postel-Vinay S, Verweij J, Demetri GD, Flaherty K, Bedard P, et al. Modifying phase I methodology to facilitate enrolment of molecularly selected patients. Eur J Cancer. 2013;49(7):1515-20.

35 Tannock IF, Osoba D, Stockler MR, Ernst DS, Neville AJ, Moore MJ, et al. Chemotherapy with mitoxantrone plus prednisone or prednisone alone for symptomatic hormone-resistant prostate cancer: a Canadian randomized trial with palliative end points. J Clin Oncol. 1996;14(6):1756-64.

36 Osoba D, Tannock IF, Ernst DS, Neville AJ. Health-related quality of life in men with metastatic prostate cancer treated with prednisone alone or mitoxantrone and prednisone. J Clin Oncol. 1999;17(6):1654-63.

37 Watson L, Qi S, Delure A, Photitai E, Chmielewski L, Smith L. Validating a patientreported outcomes-derived algorithm for classifying symptom complexity levels among patients with cancer. J Natl Compr Canc Netw. 2020;18(11):1518-25.

38 Malika N, Ogundimu O, Roberts L, Alemi Q, Casiano C, Montgomery S. African immigrant health: prostate cancer attitudes, perceptions, and barriers. Am J Mens Health. 2020;14(4): 1557988320945465.

39 Smith AB, Samuel CA, McCabe SD, Deal A, Jonsson M, Mueller DE, et al. Feasibility and delivery of patient-reported outcomes in clinical practice among racially diverse bladder and prostate cancer patients. Urol Oncol. 2021;39(1):77.e1-8. 